\title{
A New MBAC algorithm for Video Streaming based on AutoRegressive Adaptive Filtering
}

\author{
Pietro Camarda, Cataldo Guaragnella, Domenico Striccoli \\ Politecnico di Bari - Dipartimento di Elettrotecnica ed Elettronica, \\ Via E. Orabona, 4 - 70125 Bari (Italy) \\ e-mail: \{camarda, guaragnella, d.striccoli\}@poliba.it
}

\begin{abstract}
A novel strategy of Measurement Based Admission Control (MBAC) for video distribution system has been developed, aiming at obtaining the needed Quality of Service (QoS), expressed in terms of probability for the video aggregation to exceed the channel capacity. Such an algorithm is based on an AutoRegressive (AR) adaptive filter taking into account slow and quick time bit rate variations of video stream aggregation. A simple admission control strategy is presented, able to shift from the server to the client the decision about the admission of a new video stream. Simulation results, based on real MPEG encoded videos justify the application.
\end{abstract}

Keywords: admission control, linear prediction, congestion control, multimedia streaming system

\section{INTRODUCTION}

In the modern Telecommunication world, the rapid growth of multimedia transmission like Video on Demand, Distance Learning, video conferences, video telephony, etc., has renewed the research interest in this area. Quality of Service (QoS) support is necessary to guarantee multimedia delivery with low frame losses, low packet delays and jitters.

It is well known that video applications usually present Variable Bit Rate (VBR) bandwidth requirements, like the case of video sources compressed with standards (MPEG, H.263, etc). As stated in [1], VBR video traffic exhibits self-similarity and Long Range Dependence (LRD) properties that make difficult an accurate statistical analysis. On the other side, a correct video data analysis is fruitful to efficiently plan network infrastructures and Admission Control schemes.

Measurement Based Admission Control (MBAC) algorithms perform admission control by measuring actual traffic load in the network link and exploiting past measurements to estimate actual bandwidth utilization $[1][2][3]$.

In this work, we propose a new MBAC algorithm suitable for compressed video delivery with specified QoS guarantees. It establishes if a new video flow can be admitted into the network link, while respecting the QoS guarantees of the flows already running together with the new admitted flow QoS specifications. The QoS parameter taken into account is the probability for the video aggregation to exceed the channel capacity. The MBAC algorithm is based on an AutoRegressive (AR) adaptive filter taking into account slow time and quick time bit rate variations of stream aggregation, to efficiently perform video stream admission control and respect the QoS guarantees. Statistics of the stream aggregation are taken into account through past filtered measurements of bit rate aggregation.

The algorithm proposed in this work can be fruitfully applied in a context in which an aggregate behaviour analysis is crucial for admission control, like the DiffServ architecture scenario [4], in which video streaming applications often require different QoS levels according to the particular application running into the network link [5]. It has to be noted that the main disadvantage of the DiffServ architecture is that it does not provide QoS guarantees to every flow, since it establishes the behaviour of flow aggregations. This problem becomes particularly relevant for multimedia applications, that suffer from service degradations. A correct Admission Control policy is thus essential to respect the QoS guarantees for different types of multimedia applications [6].

A variety of approaches have been studied to guarantee the correct QoS level to video flows in a DiffServ context. In [5] a strategy for bandwidth allocation is proposed, for different streaming service classes. It exploits past information about streaming bit rates (the QoS metric taken into account) as feedback for bandwidth estimation, to improve QoS differentiation robustness. In [6] a novel scheduling algorithm is proposed in a DiffServ scenario for multimedia applications, to guarantee the fairness of the admitted flows at the same time obtaining an efficient performance in terms of QoS parameters (drop rate and delays among data packets). In [7] a QoS mapping is proposed for multimedia applications running into a DiffServ architecture. A QoS mapping is proposed, that is an algorithm to make a correspondence between the various kinds of data packets and the DiffServ QoS classes. In [8] the QoS issue on DiffServ networks has been applied to MPEG video traffic, treating the video flows as Assured Service (AF) flows and managing I-frames, P-frames and 
B-frames of MPEG video streams with different token buckets. Another way to improve QoS degree for video flows is to provide a constant level of perceptual video stream Quality of Service, as described in [9].

\section{THE PROPOSED SYSTEM}

In the MBAC algorithm proposed in this paper, the new flow requesting admission will be admitted and forwarded together with the rest of flow aggregation only if the QoS guarantees of every flow belonging to the aggregate (included the new one) will be satisfied.

The algorithm estimates the required bit rate margin with respect to the channel capacity, $\mathrm{C}$, considering the statistics of $\mathrm{N}$ video composite stream.

The MBAC system is based on a stream adaptive filtering technique, based on Burg linear prediction algorithm, to split the bit rate required by the composite video stream into the slow varying bit rate and its quick-time variations [3].

Statistics of $\mathrm{N}$ stream composite videos are exploited to estimate a "strict" margin with respect to the channel capacity, C, and estimate the probability of freeze for the new admitted stream in the composite video.

To make the system adaptive to the experienced multiplexed data stream input, Burg AutoRegressive (AR) estimation has been used, to exploit all the predictable information about the required bit rate. The unpredictable error has been characterized in terms of positive peak value, to be used as a measure of the margin with respect to the channel capacity to obtain a low freeze probability in the system.

Below, the proposed technique is described in detail and the measures of the channel capacity overflow probabilities are computed.

\subsection{The MBAC algorithm}

To address the problem of admission control, the statistics of an N-streams composite video have been considered. Two parameters are searched to define the MBAC algorithm: the margin with respect to the channel capacity, that for a N-streams composite video has to be used; the overflow probability for the last user accepting the limited resources video streaming system.

We have considered the statistics of both the prediction error and the predicted (low pass) composite video stream for all the cases of $\mathrm{N}$-streams composite video we can happen to manage.

Given a channel capacity $\mathrm{C}$, as in figure 1 , we have to compute a conservative but minimal margin with respect to $\mathrm{C}$, to manage all the unpredictable bit rate requirements in the $\mathrm{N}$-streams composite video.

Thus we have observed the statistics of the prediction error, first, to obtain information about the margin to be used.

The channel capacity overflow probability event depends on the union of two events:

- overflow coming from a peak in the prediction error;
- overflow coming from the peak of the required bandwidth to deliver the composite video stream (smoothed signal).

The probability of the union of these events can be approximated with the sum of the relative probabilities. As we are searching for a conservative MBAC algorithm, we can assume valid the following relation:

$$
P_{q} \approx P_{s}+P_{i} \text {. }
$$

where $\mathrm{P}_{\mathrm{q}}$ is the searched overflow probability, $\mathrm{P}_{\mathrm{s}}$ is the overflow contribute coming form the signal component and $P_{i}$ is the overflow probability coming from the prediction error, from which derives the bandwidth margin to be used due to the unpredictability of $\mathrm{N}$-stream aggregation bit rate.

As a further requirement for the MBAC system, we assume the $P_{i}$ probability be a fraction of $P_{s}$ to safely guarantee a sufficient bandwidth margin proportionally to the loss probability of the $\mathrm{N}$-stream aggregate (the established QoS parameter):

$$
P_{i}=\alpha \times P_{s}
$$

In this way, the system will be able to better absorb prediction error peaks. The choice of $\alpha$ will be established in the sequel. From (3.1):

$$
P_{q}=(1+\alpha) \cdot P_{s}
$$

With such assumptions, we can draw the graphics for the $\mathrm{P}_{\mathrm{s}}$ as a function of the bit rate for several orders $\mathrm{N}$ of the composite video stream.

By drawing the polynomial fitting curves of the bit rate as function of the $\mathrm{P}_{\mathrm{s}}$, we can compute the bit rate of the composite video signal as a function of the overflow probability $\mathrm{P}_{\mathrm{s}}$.

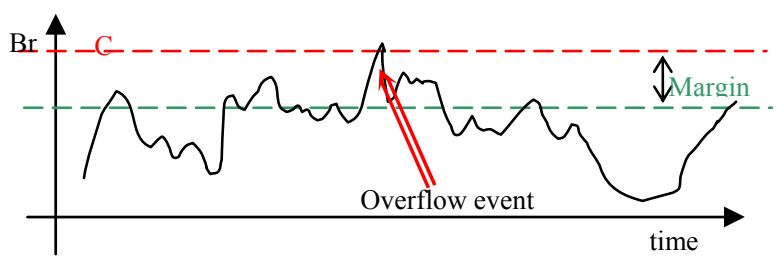

Figure 1: bit rate time evolution and definition of the channel capacity and margin.

\subsection{The MBAC implementation}

The overflow probability can be used to define a MBAC system; the N-streams composite video global bit rate has to be considered; the two components bit rates respectively coming from the unpredictability of the bit rate and from the smoothed version of the bit rate time series have to be considered.

Two different measures have been carried out, one over the predicted signal and the other over the prediction error. The first addressed system parameter to define is the extension of the observation window to be used in this application. The second, the filter taps to be used in the LP filter. Simulations have shown that a fair choice of such parameters is 1000 samples for the processing window (corresponding to about 30 seconds at $30 \mathrm{fps}$ ), and a LP 
filter of 15 taps. In all the simulation results here presented such values have been used.

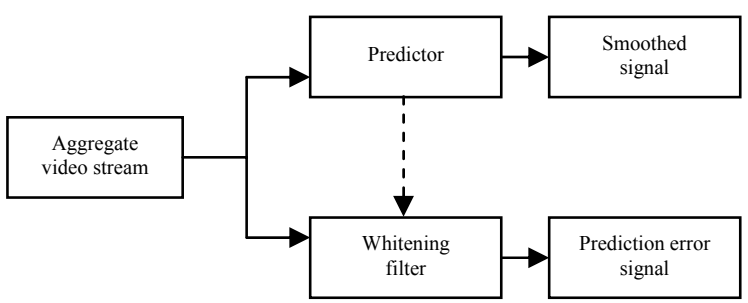

Figure 2: Processing scheme

Let $f_{n}(\mathrm{~b})$ be the probability density function (pdf) of the prediction error with respect to the bit rate, and $F_{n}(\mathrm{~b})$ its corresponding cumulative distribution, the quantity $F_{c n}(\mathrm{~b})=1-F_{n}(\mathrm{~b})$ represents the probability that the event "the difference between estimated bit rate and the true one is grater than b". This probability will be used in the proposed MBAC algorithm.

To accept a new streaming client, the available composite video bit rate must satisfy the relation:

$$
B R\left(N+1, P_{s}\right)+\Delta B r\left(P_{s} / \alpha\right) \leq C
$$

where $B R$ and $\Delta B r$ are evaluated by (3.5). BR represents the composite video stream bit rate in the hypothesis the new stream is accepted and multiplexed together with the given $\mathrm{N}$-streams composite video, to originate a $(\mathrm{N}+1)$ streams composite video as a function of the overflow probability Ps, and $\Delta B r$ is the corresponding margin with respect to the channel capacity $\mathrm{C}$ to absorb the peaks of the BR due to the unpredictability of the phenomenon. As previously stated, $P_{i}=P_{s} / \alpha$ has to be littler than $P_{s}$, but not excessively to avoid an over-conservative admission control policy, that would tend to reject flows even if there are sufficient bandwidth requirements. A good choice is to choose $P_{i}$ an order of magnitude littler than $P_{s}$, so we choose for our experiments $\alpha=0.1$.

The curves of the bit rate probability obtained with all $\mathrm{N}$ streams composite videos for the prediction error tend to saturate, as $\mathrm{N}$ increases, to the curve in figure 4 . To be conservative and make the algorithm simple, we have considered a single curve corresponding to $\mathrm{N}$ large.

Whenever $B R+\Delta B r<C$ the new client will be admitted to the service, otherwise the probability of overflow will be estimated and passed to the client that, considering such information, might decide to accept the service or deny it. The limit condition (equality) can be assumed in (3.4).

For each N, and defined $\alpha$ in (3.2), the complementary curves of the probability as a function of the bit rate can be approximated by polynomial functions in the variable $\mathrm{P}_{\mathrm{s}}$; we compute a single polynomial function for the unpredictability of the bit rate, $\Delta \mathrm{Br}$, and a family of curves for the $\mathrm{N}$-streams cases of composite video streams.

Thus we have:

$$
\left\{\begin{array}{l}
\Delta B r\left(P_{i}\right)=\Delta B r\left(P_{s} / \alpha\right)=\sum_{n=0}^{L} c_{n} \cdot P_{s}^{n} \\
B R\left(N, P_{s}\right)=\sum_{n=0}^{L} a_{n}(N) \cdot P_{s}^{n}
\end{array}\right.
$$

Assuming to be in proximity of the channel capacity, with a $\mathrm{N}$-streams composite video, the algorithm steps consist in:

I. Compute the probability $\mathrm{P}_{\mathrm{s} 0}$ due to the $(\mathrm{N}+1)$ case finding the zero of the polynomial equation $\mathrm{BR}\left(\mathrm{P}_{\mathrm{s} 0}\right)=\mathrm{C}$

$$
C=B R\left(N+1, P_{s 0}\right)=\sum_{n=0}^{L} a_{n}(N+1) \cdot P_{s 0}^{n}
$$

II. Compute the margin with respect to the channel capacity for the $(\mathrm{N}+1)$-streams composite video, $\Delta \mathrm{Br}\left(\mathrm{P}_{\mathrm{s} 0}\right)$ :

$$
\Delta B r\left(P_{s 0} / \alpha\right)=\sum_{n=0}^{L} c_{n} \cdot P_{s 0}^{n}
$$

III. Compute the new maximum bit rate to be assumed for the smooth signal, $\mathrm{BR}_{1}$ :

$\mathrm{BR}_{1}=\mathrm{C}-\Delta \mathrm{Br}\left(\mathrm{P}_{\mathrm{s}} / \alpha\right)$

IV. Compute the minimum value assumed at the new computed bit rate for the $(\mathrm{N}+1)$ case, $\mathrm{P}_{\min }$, as the solution of the polynomial equation $\mathrm{BR}_{1}=$ $\mathrm{BR}\left(\mathrm{N}+1, \mathrm{P}_{\min }\right)$ :

$$
B R_{1}=B R\left(N+1, P_{\min }\right)=\sum_{n=0}^{L} a_{n}(N+1) \cdot P_{\min }^{n}
$$

V. Compute the estimated overflow probability as:

$$
P_{q}=(1+\alpha) \cdot P_{s}
$$

\section{EXPERIMENTAL RESULTS}

The experimental results we present all refer to real data of composite videos obtained statistically multiplexing MPEG encoded YUV-CIF videos, with GOP of 12 frames and $30 \mathrm{fps}$. All the created composite video streams have been obtained randomly mixing sources with different statistics, and composite videos are limited to 20 sources composite video.

Figure 3 refers to the obtained complementary cumulative distribution function for the cases of $\mathrm{N}$ multiplexed sources, with $\mathrm{N}$ in the set $[1,20]$.

Curves in figure 3 and the curve in figure 4 have been polynomial fitted to give analytical expression in (3.5).

Two sets of N-streams composite videos time series bit rates have been produced: a training one, used to obtain curves in figures 3 and 4 , and a test set, used to obtain experimental results.

Polynomials fitting curves for such functions have been estimated, as described in section 3.2.

The test of the proposed algorithm has been carried out on the test set of $\mathrm{N}$-streams composite videos to estimate the expected overflow event.

A capacity $\mathrm{C}$ communication channel has been assumed, and a number of video, $\mathrm{N}$, in the composite video stream has been selected in order be close to the channel capacity and give a negligible overflow probability. A request of service by a $(\mathrm{N}+1)$-th client has been assumed, and the 
overflow probability for that client has been estimated as described in section 3.2.

Repeated experiments in the same conditions have been carried out. The experimental measure of the overflow probability for the test cases have been computed as the fraction between the overflow experienced time duration and the total duration of the $(\mathrm{N}+1)$-streams composite videos.

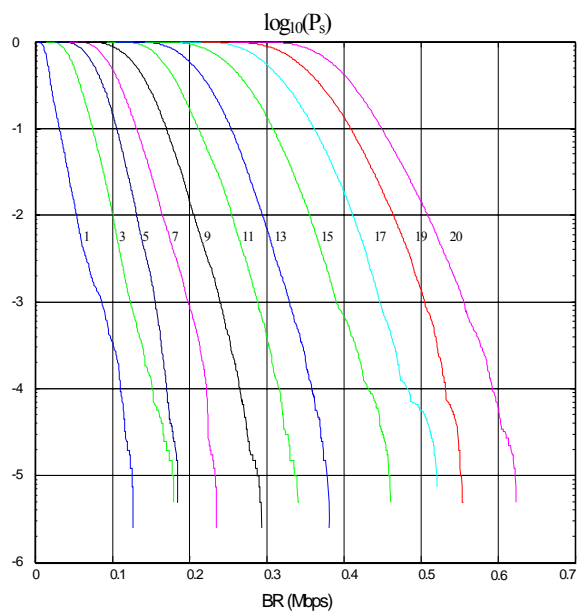

$\mathrm{Fi}$

gure 3: $1-\mathrm{F}_{\mathrm{s}}(\mathrm{BR})$ : smoothed complementary cumulative probability functions of the bit rates for several orders of composite videos.

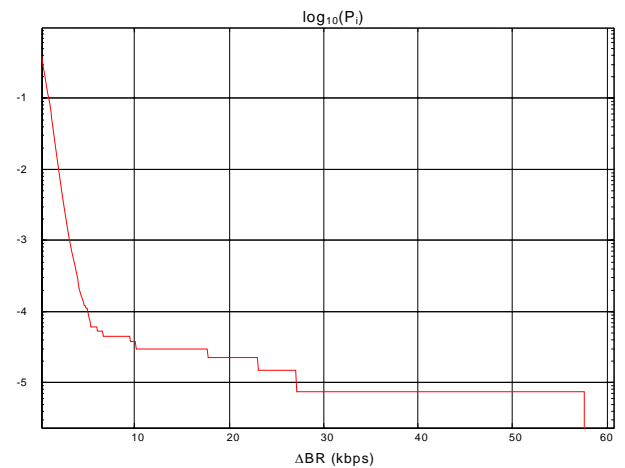

Figure 4: $1-\mathrm{F}_{\mathrm{n}}(\mathrm{BR})$ : unpredictability cumulative complementary probability functions of the bit rates for several orders of composite videos.

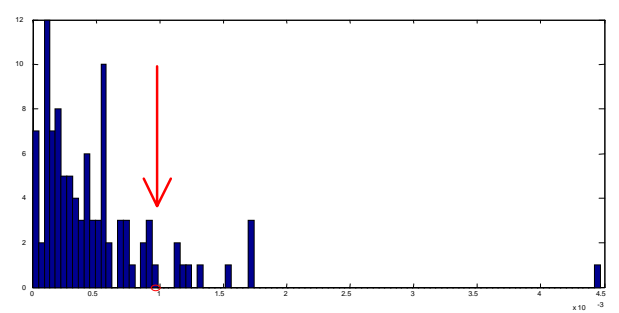

Figure 5: Histogram of tested sequences: signalled by an arrow, the point on the horizontal line represents the estimated overflow probability; the estimated probability is lower of the experienced one only for the $10 \%$ of the total test cases.
The obtained overflow probability is mostly well predicted by the proposed algorithm.

Figure 5 presents the histogram of the obtained probability values for 100 cases of $(\mathrm{N}+1)$-streams composite videos in the test set. The fraction of test cases showing an experienced overflow probability greater than the estimated one results lower than $10 \%$ in our simulations; also, the difference in probability value is never much higher than the estimated one.

\section{CONCLUSIONS AND FUTURE WORK}

In this work a measure based predictive admission control algorithm has been introduced.

The main advantage with respect to actual MBAC systems is the ability of predicting the performance the video streaming server is able to obtain in a given traffic congestion situation, and leave the client the decision about accepting the per-flow estimated QoS the system is experiencing, or deny the service.

Good performance in terms of predicted probability have been obtained.

The proposed technique will be straightforwardly generalized to heterogeneous traffic classes (audio, video, VoIP, music, data), the goal of a future work.

\section{REFERENCES}

[1] M.W.Garrett, W.Willinger, "Analysis, Modeling and Generation of Self-Similar VBR Video Traffic", ACM Computer Communications Review, Vol.24, N.4, pp.269-280, October 1994.

[2] M.Crovella, A.Bestavros, "Self Similarity inWorld Wide Web Traffic: Evidence and Possible Causes" In Proc. ACM Sigmetrics '96, pp.160-169, May 1996.

[3] M.Glossglauser, D.Tse, "A time-scale decomposition approach to Measurement Based admission control", In Proc. IEEE Infocom, pp.1539-1547, March 1999.

[4] S.Blake, D.Black, M.Carlson, E.Davies, Z.Wang, W.Weiss, "An Architecture for Differentiated Services", IETF RFC-2475, December 1998.

[5] X.Zhou, C.-Z.Xu, "Analysis of a Bandwidth Allocation Strategy for Proportional Streaming Services", in Proc. IEEE International Conference on e-Commerce Technology, pp.373-376, July 2004.

[6] D.Q.Hai, S.T.Vuong, "Dynamic-Distributed Differentiated Service for Multimedia Applications", in Proc. International Conference on Dependable Systems and Networks, pp.586-594, June 2000.

[7] F.Zhang, M.R.Pickering, M.R. Frater, J.F.Arnold, "Optimal QoS Mapping for Streaming Video over Differentiated Services Networks", in Proc. IEEE International Conference on Acoustics, Speech, and Signal Processing, Vol.5, pp.744-747, April 2003.

[8] N.E.Rikli, M.Al-Abdulmunem, "MPEG Traffic over Diffserv Assured Service", in Proc. $9^{\text {th }}$ Asia-Pacific Conference on Communications, Vol.2, pp.494-498, September 2003

[9] D.Quaglia, J.Carlos de Martin, "Adaptive Packet Classification for Constant Perceptual Quality of Service Delivery of Video Streams over Time-Varying Networks", in Proc. 2003 International Conference on Multimedia and Expo, Vol.3, pp.369-372, July 2003. 